\title{
La Nanoelectrónica y la Electrónica Molecular
}

\author{
Jorge M. Seminario and Karim Salazar \\ Department of Chemical Engineering \\ Department of Electrical and Computer Engineering \\ Department of Materials Science and Engineering \\ Texas A\&M University \\ College Station, Texas, USA \\ E-mail: seminario@tamu.edu
}

Recibido el 10 de diciembre del 2016; aceptado el 18 de diciembre del 2016

DOI: https://doi.org/10.33017/RevECIPeru2016.0007/

\section{Resumen}

La nanotecnología ofrece herramientas para el diseño de sensores automatizados capaces de neutralizar agentes tóxicos mediante el desarrollo de operaciones lógicas y comunicación de la información. El uso de componentes moleculares en la fabricación de nanosensores, resulta ventajoso debido a la alta capacidad de transmisión de información, eficiencia y rapidez de las operaciones. El objetivo es desarrollar nanosensores inteligentes mediante operadores lógicos en base a la interacción del potencial electrostático molecular y el transporte de información mediante señales vibracionales. El presente trabajo muestra resultados preliminares basados en principios básicos de la materia, como la mecánica cuántica, mecánica molecular y dinámica molecular.

Las cascadas de compuertas lógicas moleculares operarían a través de señales basadas en el potencial electrostático molecular. Permitiendo la realización de funciones matemáticas de todo tipo mediante arreglos de compuertas lógicas. Siendo los valores positivo y negativo del potencial electrostático señales de entrada y salida de una compuerta lógica. Finalmente, la información contenida en la salida seria transportada mediante fluctuaciones de los modos vibracionales a través de moléculas lineales en el rango de terahertz $(\mathrm{THz})$.

Descriptores: Nanotecnología, nano-sensores programables, compuertas lógicas moleculares, potenciales electrostáticas moleculares, transporte eléctrico, señales vibracionales.

\begin{abstract}
Nanotechnology offers tools for the design of automated sensors capable of neutralizing toxic agents through the development of logical operations and communication of information. The use of molecular components in the manufacture of nanosensors is advantageous because of the high capacity of information transmission, efficiency and speed of operations. The goal is to develop intelligent nanosensors using logical operators based on the molecular electrostatic potential interactions and the transport of information through vibrational signals. The current work shows preliminary results based on basic principles of matter, such as quantum mechanics, molecular mechanics and molecular dynamics.

The cascades of molecular gates would operate through signals based on molecular electrostatic potential. These logical gate arrays will allow us to perform all kind of mathematical functions. The positive and negative values of the molecular electrostatic potential will be the input and output signals of a logic gate. Finally, the information contained in the output will be transported through linear molecules by fluctuations of vibrational modes in the terahertz $(\mathrm{THz})$ range.
\end{abstract}


Keywords: Nanotechnology, programmable nano-sensors, logic gates, molecular electrostatic potential, electron transport, vibrational signals.

\section{Introducción}

El desarrollo de tecnologías de ensamblaje así como las técnicas de deposición y manipulación a nivel molecular abren un amplio panorama en el diseño y fabricación de circuitos y dispositivos moleculares [1-4]. La capacidad de decidir la neutralización de un agente tóxico detectado, mediante operaciones lógicas basadas en señales vibracionales [5], potencial electrostático molecular [6], orientación del spin electrónico [7-9] y bi-estabilidad óptica [10-12], resultan en novedosos panoramas de codificación y decodificación de información, aplicables para circuitos en la escala de nanometros. Por otro lado, el procesamiento lógico de información de respuestas bioquímicas, resulta importante en el diseño de sensores, ya que las rutas metabólicas son altamente controladas y especificas [13]. Esta nueva visión de sensores inteligentes requiere del estudio de las interacciones moleculares de los componentes de nanoarquitecturas, los cuales difícilmente se logran estudiar experimentalmente; sin embargo, se logra simular escenarios hipotéticos mediante la mecánica quántica, mecánica molecular y dinámica molecular [14].

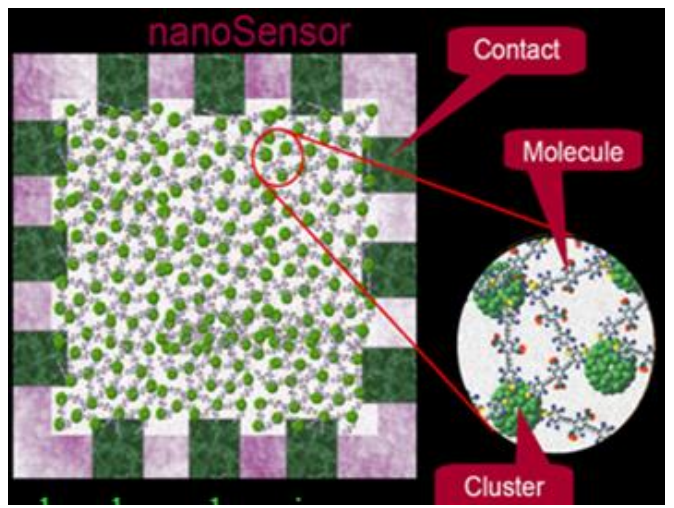

Figura 1. Bosquejo de un sensor inteligente capaz de procesar información de algún agente biológico detectado.

Nuestra investigación se centra en el diseño y estudio de los componentes e interacciones de nano-celdas programables [15-17] (Figure 1) a través de procesos computacionales para fines de detección de agentes químicos y biológicos [18]. Los componentes son analizados a nivel molecular mediante herramientas usadas en nanotecnología, a fin de analizar el proceso de detección molecular de los componentes sensibles del sensor, así como la comunicación de la información mediante moléculas portadoras y la traducción del fenómeno químico a señales eléctricas y vibracionales fácilmente detectables. En este contexto, se ha desarrollado diversos escenarios para ensamblar eficientemente cada componente del nano-detector a fin de conferirle especificidad, rapidez y lógica. Los escenarios son (1) potencial electrostático molecular, (2) señales vibracionales y (3) transporte de electrones a través de moléculas, tanto las que intervienen directamente en la detección como las que forman parte de los demás componentes del sensor. Cabe resaltar que la mayoría de sensores están basados en la medición de la variación de la corriente eléctrica; sin embargo el uso del potencial electrostático molecular y señales vibracionales resulta muy eficiente debido al bajo poder de consumo y largo ancho de banda, el cual nos permite operar en el rango de $\mathrm{THz}$ para codificar, procesar y detectar la información con gran rapidez.

\section{Potencial electrostático molecular (PEM).}

Las interacciones moleculares pueden codificarse mediante el cambio del PEM de un sistema molecular. En algunos casos, el MEP de un compuesto es inducido por la presencia de otro en tal medida que se obtienen operaciones binarias lógicas, permitiéndose diseñar circuitos capaces de realizar operaciones lógicas en cascada a nivel molecular [6, 19, 20] (Figura 3). Mediante el uso de la mecánica cuántica, hemos diseñado diversas compuertas lógicas compuestas por moléculas, en donde el potencial electrostático molecular se representa como un dígito binario, si es negativo un 0 y si es positivo un 1. Por ejemplo, encontramos una compuerta AND como resultado de la interacción del potencial electrostático positivo y negativo de dos moléculas de cianuro de hidrogeno sobre el 3- y 5-fluor del 1,3,5-trifluorobenzene (TFB). El output se lee en la vecindad de un átomo de berilio ubicado a $3.2 \AA$ de TFB, sobre el 1-fluor [19]. Nuestros estudios muestran la posibilidad de diseñar un dispositivo molecular con función de compuerta lógica OR compuesto por 1,1-diethynylethene y dos moléculas de agua como inputs, en donde la operación lógica se obtiene como resultado de la aplicación del potencial electrostático molecular positivo y negativo de las dos moléculas de agua sobre el grupo ethynyl ubicados en los extremos del compuesto 1,1-diethynylethene, mientras que el ethynyl grupo ubicado en la superficie es usado como región del output (Figura 2). En estos dos 
casos, las señales de entrada se obtienen de moléculas con potenciales electrostáticos moleculares positivo y negativo bien establecidos, como el agua o el cianuro de hidrogeno, pudiendo usarse otras que resulten más fácilmente manipulables. Es interesante mencionar que en todos los casos analizados, la energía para ingresar la información o activar la compuerta molecular, en el peor de los casos corresponde a $7 \mathrm{kcal} / \mathrm{mol}$ [21]. Esto es una cantidad de energía por lo menos tres a cuatro órdenes de magnitud menor que la necesaria en dispositivos semiconductores.

Hemos analizado también el efecto del sustrato en la compuerta lógica OR compuesta por 1,1diethynylethene y dos moléculas de agua como entradas, en donde los elementos de la compuerta molecular están separados $4 \AA$ del sustrato. En este estudio se encontró que el grafeno afecta en la lectura del potencial electrostático molecular en la vecindad de la salida cuando se aplica el potencial electrostático molecular negativo de dos moléculas de agua ya que se forma un potencial negativo alrededor del 1,1-diethynylethene debido al potencial negativo que el grafeno muestra en su superficie; sin embargo el efecto se neutraliza cuando se agrega más capas de grafeno como sustrato. El silicón sin embargo no influye en la compuerta lógica debido al bajo potencial mostrado [22].

Para la aplicabilidad de las compuertas lógicas en base al PEM, es necesario decodificar el fenómeno mediante el diseño de micro-interfaces. Nuestro estudio muestra la lectura de una compuerta lógica OR mediante la medida de la corriente electrónica de un sistema compuesto por 3,6-bis(phenylethynyl) conectado a dos electrodos de oro mediante el enlace químico con sulfuro, en el cual dos átomos de carbono del ring central son reemplazados por átomos de nitrógeno a fin de generar un momento dipolar necesario para la rotación del anillo, ante la aplicación un potencial electrónico externo inducido por el potencial electrostático molecular de la compuerta lógica OR en la vecindad de la región del output. Los resultados muestran que la rotación del anillo central genera una diferencia en la corriente electrónica a través de la molécula [23].

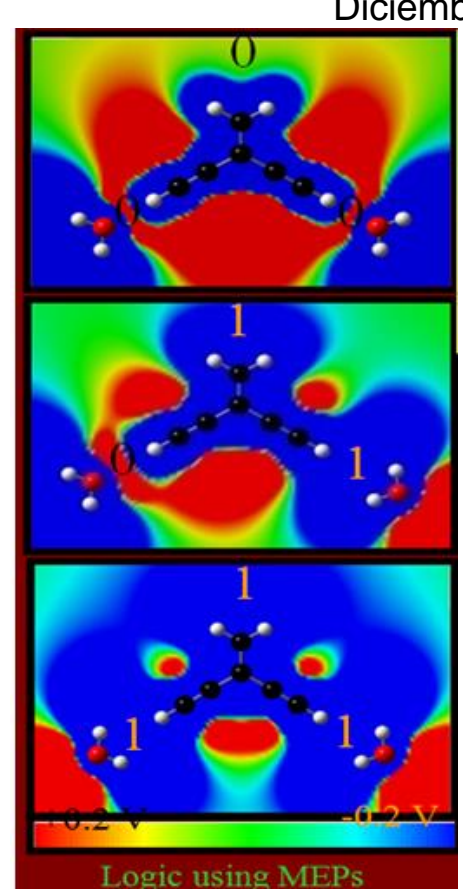

Figura 2. Compuertas lógica moleculares basadas en el potencial electrostático molecular. El grafico muestra a una compuerta OR que está compuesta por un 1,1-diethynylethene $y$ dos moléculas de agua (input).

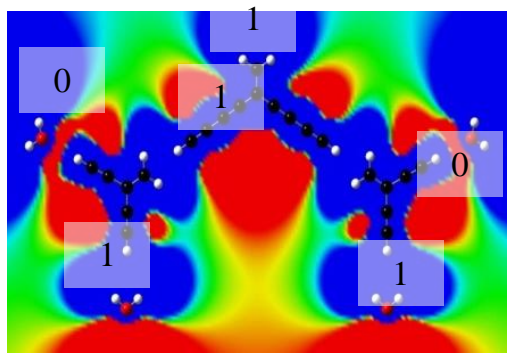

Figure 3. Compuertas lógicas en cascada en los cuales las entradas son las salidas de dos compuertas OR, cada una compuesta por 1,1diethynylethene y dos moléculas de agua usadas como potenciales de entrada. Todas las combinaciones de entrada fueron probadas exitosamente.

\section{Señales vibracionales.}

Nuestros estudios demuestran que los modos vibracionales de un analito se logran imprimir sobre el clúster de silicón o capas de grafeno fácilmente detectables, permitiendo la detección de pequeñas concentraciones de analito mediante el análisis de espectroscopia sobre la superficie de materiales específicos y clústeres [24, 25]. Para ello, se analizó la interface de un clúster de silicón con un grupo bencénico, a fin de identificar la impresión de modos vibracionales de la molécula blanco en el espectro infra-rojo del clúster. El clúster de silicón fue pasivado con 
átomos de hidrogeno, su forma es piramidal y el benceno se encuentra unido al clúster a una distancia de $1.90 \AA$ en dos puntos de estudio, en el ápice de la pirámide y por debajo de la base de la misma [26].

En el caso del grafeno, la interacción molecular de gases adsorbidos sobre la superficie de varias capas del material, actúa como un amplificador de señales vibracionales en la región de $\mathrm{THz}(<66$ $\mathrm{THz}$ ). Se analizó moléculas como $\mathrm{N}_{2}$ y $\mathrm{O}_{2}$, a modo de prueba, adsorbidas sobre la superficie grafeno con una distancia de interface de $3.50 \AA$; para los sistemas compuestos por tricapas de grafenos, se usó el posicionamiento de Bernal $(A B A B)$ y rombohedral $(A B C A B C . .$.$) . El estudio$ de la espectroscopia Raman de los sistemas de multi-capas de grafeno descritos, muestra intensidades en la región del $\mathrm{THz}$ creados debido a la interacción molecular entre ellas, en donde los modos vibracionales de las moléculas gaseosas $\mathrm{N}_{2}$ y $\mathrm{O}_{2}$ se imprimen, aumentando el número de modos e intensidad [27].

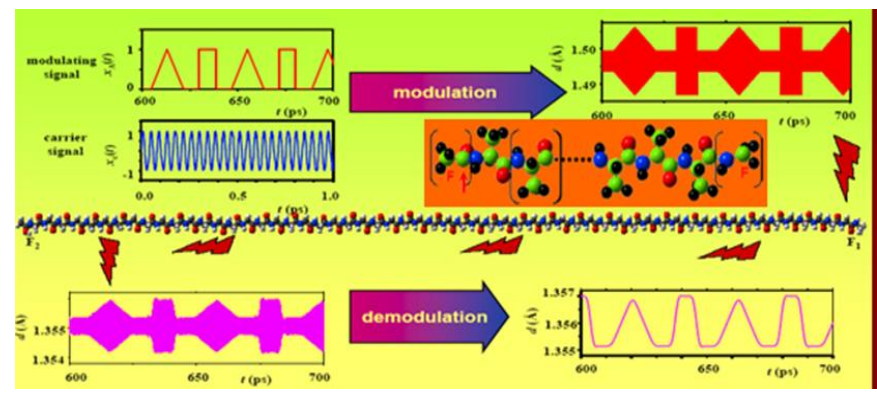

Figura 4. El envío de señales a través de biomoléculas lineales fue probado mediante simulaciones de dinámica molecular. Una señal modulante modula a una portadora de frecuencia similar a un de los modos vibracionales de la molécula lineal. La señal introducida en un terminal del cable molecular viajo a través del cable y fue recuperada en el otro terminal después de su demodulación la señal de salida fue prácticamente similar a la entrada [28, 29].

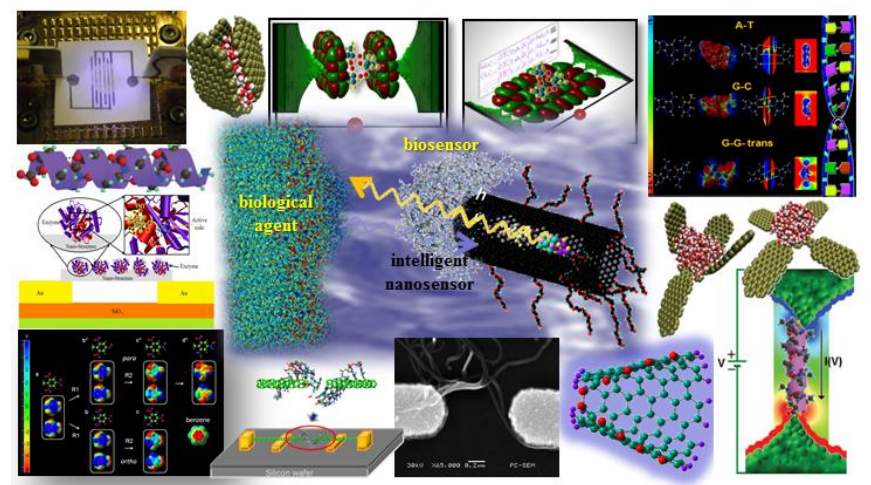

Figura 5. Alternativas para la implementación de sensores inteligentes capaces de buscar agentes biológicos específicos.

Nuestros estudios muestran que las señales vibracionales se transmiten sobre moléculas lineales muy largas como poliglicina y polialanina. Para este estudio se usó dinámica molecular y técnicas de procesamiento de señal digital (Figura 4). Para el caso de poliglicina compuesta por 1000 monómeros, se demostró que la inyección de señales vibracionales a una frecuencia modulada sobre un enlace $\mathrm{C}-\mathrm{N}$, se transmite sobre un extremo hacia el otro de un polímero. La molécula de poliglicina se conectó a clústeres de oro en ambos extremos mediante el enlace con sulfuro, a modo de estudiar el uso de moléculas como carriers de señales vibracionales en rango de $\mathrm{THz}$, modulándolas a través de ellas a frecuencias ubicadas en la región del infrarrojo. Los resultados obtenidos de las señales vibracionales sobre diversos puntos de la molécula varían; por ejemplo el enlace C-N manipulado por la inyección de una señal de 1.8 $\mathrm{THz}$ de banda ancha, muestra un pico en la frecuencia de $23.81 \mathrm{THz}$ sobre el extremo expuesto, luego a lo largo de la molécula decae con una tasa del $6 \%$ de la señal por residuo [5]. También se ha estudiado la molécula lineal polialanina, como línea de transmisión para alimentar el potencial electrostático molecular de un dispositivo OR lógico molecular, mediante la transferencia de señales vibracionales desde un extremo a otro de la polialanina. El input es un pulso con forma determinada, introducido mediante vibraciones mecánicas sobre el enlace C-C desde un extremo de la molécula y el output es tomado mediante la oscilación del enlace $\mathrm{N}-\mathrm{C}$ ubicado en el extremo opuesto [30]. 

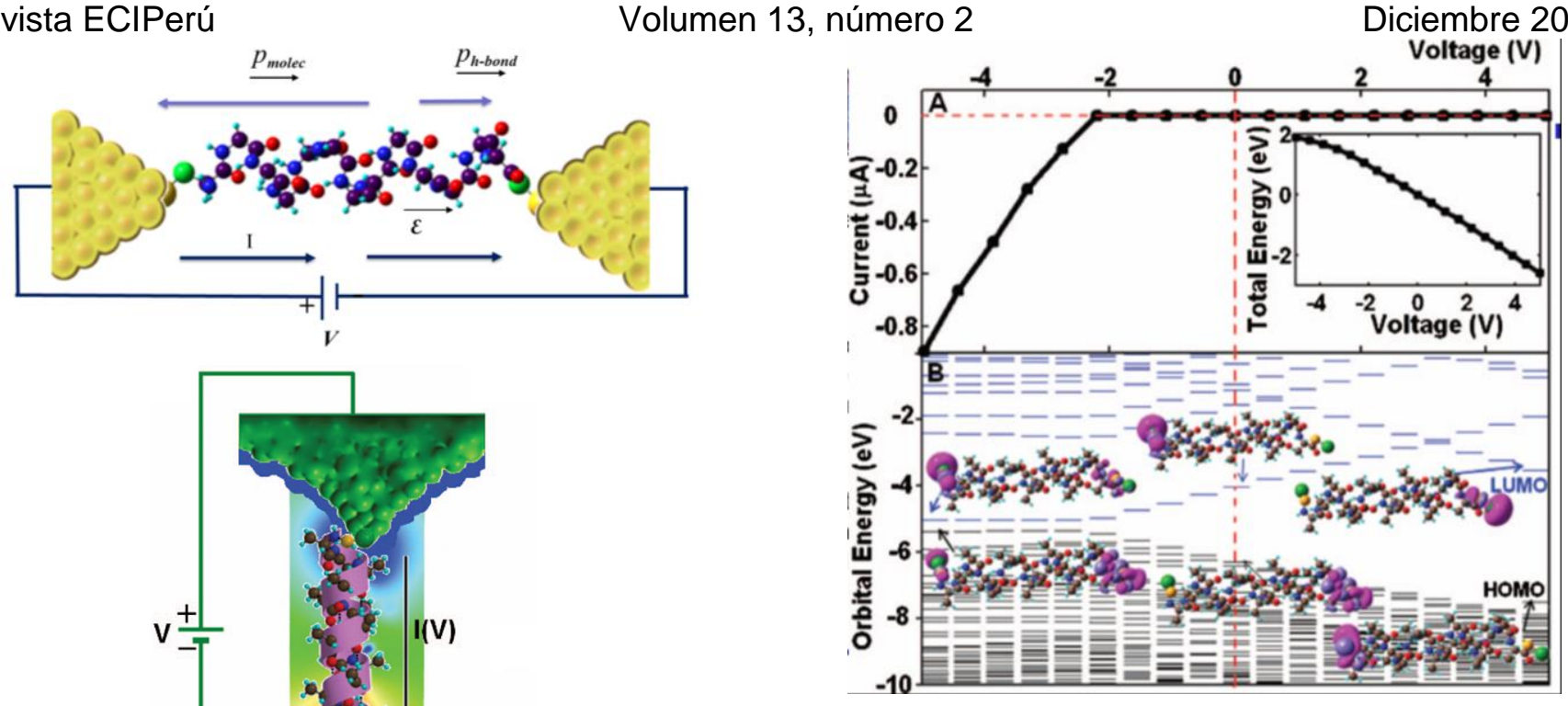

Figura 7. Parte A muestra la curva corriente versus voltaje de la molécula de polyalanina la cual se comporta como un diodo perfecto. Con voltajes positivos la molécula no conduce corriente (es un aislante) pero con voltajes menores de 2 voltios, la molécula se comporta como un conductor. La parte $B$ de la Figura

Figura 6. Los diodos son los más básicos dispositivos electrónicos y pueden ser usados como sensores de solo un nanómetro de largo y solo unos Angstroms de diámetro. Es realmente extraordinario como la naturaleza ya los había implementado en seres biológicos de una manera tan perfecta para que puedan funcionar en circuitos de tres dimensiones.

Para el caso del reconocimiento molecular de sistemas biológicos, se analizó la espectroscopia de dicroísmo circular (DC) para el sitio de activación de la enzima soluble guanilate ciclase, el cual consiste en la formación de un enlace covalente del $\mathrm{N}$ y $\mathrm{C}$ de los gases $\mathrm{NO}$ y $\mathrm{CO}$ respectivamente con el ion ferroso del complejo formado por el anillo porfirina e histidina localizados en la enzima. En este estudio se comparó diversas formas de traducir el reconocimiento molecular del modelo biológico como corriente-voltaje, espectro infrarojo, Raman y DC. EI DC espectro resulto más eficiente que el infrarojo, Raman y la respuesta eléctrica del sistema; debido a la diferencia del ángulo que se forma con el enlace generado durante la detección ( $\mathrm{Fe}-\mathrm{CO}$, $\mathrm{Fe}-\mathrm{NO}$ y $\mathrm{Fe}-\mathrm{O}_{2}$ como posible interferencia) con respecto al plano de la porfirina, obteniéndose una respuesta negativa para el $\mathrm{CO}$ y positivas para el $\mathrm{NO}$ y $\mathrm{O}_{2}$, sin embargo los dos últimos casos presentan características opuestas [31]. muestra la explicación de porqué este comportamiento tipo diodo. Con voltaje cero, la energía entre el orbital molecular ocupado más alto (HOMO) y el orbital molecular desocupado más bajo (LUMO) es muy alta que no permite la conducción de electrones. Si el voltaje aplicado se incrementa, hace que la separación HOMOLUMO (HLG) se incremente, deteniendo aún más el paso de la corriente de electrones. Sin embargo, cuando el potencial aplicado se hace negativo, el HLG se reduce hasta que el HOMO y el LUMO colapsan permitiendo el paso de electrones. Lo más saltante de esta demostración es que la corriente eléctrica pasa a través de los puentes de hidrogeno que puentean a la hélice de la alamina que en este caso tiene la función de soporte del dispositivo.

Bajo este escenario, es posible transmitir la señal de un ligando durante el reconocimiento de la proteína receptora, el cual causa cambio configuracionales propagándose desde el sitio de afinidad hacia la superficie $y / 0$ extremo de la proteína, mediante las interacciones de noenlaces. 


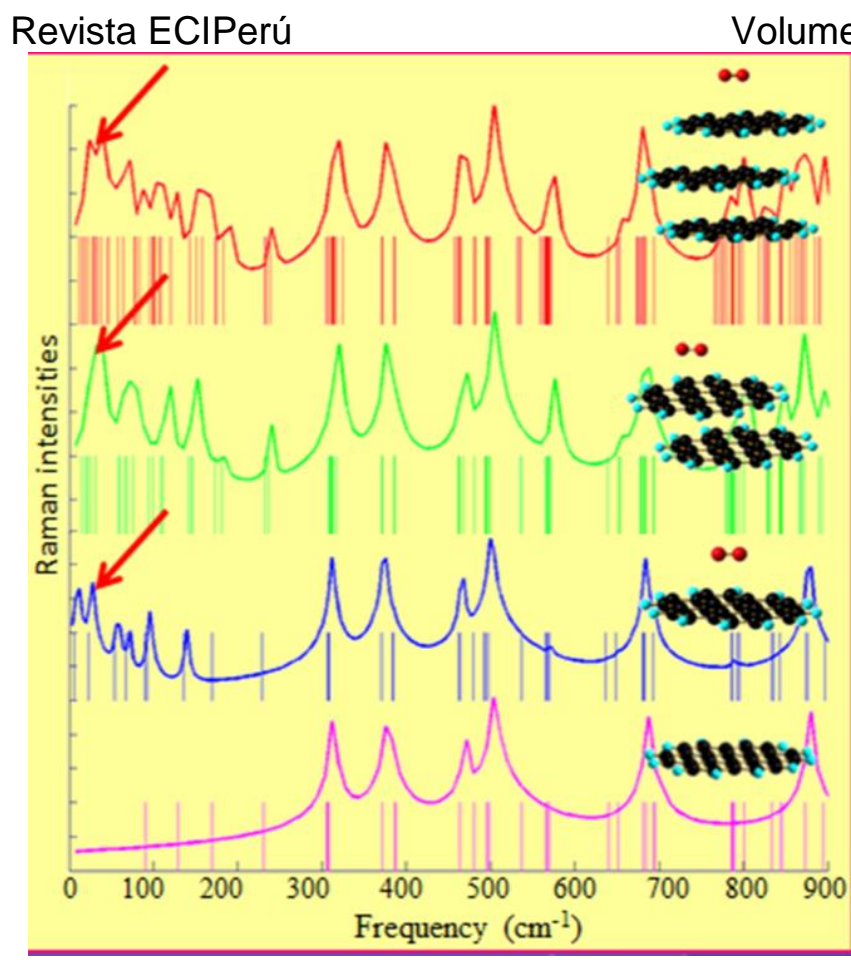

Figura 9. Moléculas de grafeno son capaces de mostrar plasmones que no son más que electrones en estado de plasma. Esto se debe a la alta resonancia de los orbitales moleculares $s p^{2}$. Este plasma puede vibrar de manera diferente con cualquier molécula que se acomode en la superficie y que por más pequeña que sea, será detectable en la molécula de grafeno. Esto permitirá en un futuro cercano la fabricación de sensores muy específicos y precisos. Grafenos son también candidatos favoritos para desarrollar una electrónica post-CMOS. La figura muestra el espectro de Raman cuando una molécula está presente y cuando no lo está sobre la molécula de grafeno.

\section{Transporte de electrones}

La probabilidad del transporte de electrones de una molécula conectada a nano-electrodos se resuelve mediante la teoría de densidad funcional y ecuaciones de Green. Nosotros aplicamos estas técnicas para predecir las características electrónicas de un sistema, el cual puede ser el mismo fenómeno a detectar, como la secuencia de nucleótidos erradas en el DNA; así como moléculas e interfaces de los componentes electrónicos del nano-sensor. Para la lectura de secuencias de nucleótidos, nuestras investigaciones concluyen que las características eléctricas son identificables durante la medición transversal de la corriente, simulando el paso vertical y controlado del DNA dentro de un poro con nano-electrodos de oro en su interior [32, 33]. Errores de complementariedad de los nucleótidos como AA, CC, GG, TT resultan menos
Diciembre 2016 conductivos que los pares complementarios de nucleótidos, siendo el más conductor el par CT [33]. Se realizó simulaciones de la aplicación de corriente eléctrica transversal al DNA, considerando un bulk de átomos de oro y de moléculas de DNA como electrodos, resultando más conductivo cuando se considera un bulk de oro como electrodo. La presencia de iones de $\mathrm{Na}$ como contra-iones sobre el grupo fosfato causa el incremento en las características del sistema a comparación del $\mathrm{H}+$.

Las demás partes del componente del sensor evaluados son: los conectores, switches de activación, clústeres y su interacción con el sustrato. El estudio de la conducción electrónica de nanotubos de carbono metálicos $(4,4)$ como conector entre oligo(phenylene ethylene) y clúster de silicón, indica que existe mayor conducción cuando el plano de los anillos de la órganomolécula se encuentran con una configuración coplanar en comparación con la configuración perpendicular. Se analizó también diversos estados de oxidación para el sistema (neutral, anión, di-anión y tri- anión), siendo más conductivo en condición neutral. En el caso de switches moleculares, se ha analizado la fotoconverción de la provitamina D3 a vitamina D3, por medio del cual un pequeño pulso de laser UV representa una alternativa para introducir señales a sistemas moleculares. La simulación y medición en experimentos del reactante y producto de la fotoconverción resulta identificable, siendo mayor la conducción de la vitamina D3 [34].

\section{Conclusiones}

El diseño de nano-sensores programables mediante el ensamble de componentes a escala nano, resulta viable a través de las operaciones lógicas obtenidas como resultado de la interacción del potencial electrostático molecular de un sistema compuesto por moléculas, en el cual los valores positivo y negativo del potencial molecular se representan como dígitos binarios 1 y 0 , respectivamente. Otros indicios de la viabilidad del diseño de nano-sensores autómatas son; (1) la operatividad de la compuerta lógica molecular cuando se encuentra adsorbido sobre superficies inertes como los del clúster de silicón y bicapa de grafeno, (2) la traducción del potencial electrostático molecular de un nano-sistema a mediciones aplicables a escala micro, como la corriente eléctrica de un sistema compuesto por moléculas atrapadas en nano-electrodos de oro mediante enlaces sulfuro, cuyo principio 
metodológico se basa en el cambio configuracional de moléculas orgánicas ante la interacción del campo eléctrico generado por potenciales electrostáticos de un sistema externo; (3) fácil funcionalización de moléculas orgánicas a nano-estructuras metálicas como el nanotubo de carbono $(4,4)$, el cual es el nanotubo con diámetro más pequeño sintetizado; (4) la capacidad de moléculas orgánicas lineales (poliglicina) para transportar la inyección de vibraciones mecánicas moduladas en el rango de $\mathrm{THz}$ desde un extremo hacia el otro, el cual puede alimentar el potencial electrostático molecular de una compuerta lógica; (5) la especificidad y el fácil discernimiento de la detección a través de la espectroscopia durante el reconocimiento molecular realizadas por biomoléculas y (6) la generación de señales vibracionales en orden de $\mathrm{THz}$ de multi-capas de grafeno y la impresión de modos vibracionales de compuestos adsorbidos.

\section{Agradecimientos}

Agradecemos a los miembros presentes y pasados de nuestro grupo de investigación de Molecular y Nano Electrónica. También agradecemos a la U.S. Army Research Office, U.S. Defense Threat Reduction Agency y al U.S. Department of Energy/Basic Energy Science por el financiamiento de nuestras investigaciones.

\section{Referencias}

[1] J. Brugger, "Nanotechnology impact on sensors," Nanotechnology, vol. 20, p. 430206, 2009.

[2] S. Y. Quek, M. Kamenetska, M. L. Steigerwald, H. J. Choi, S. G. Louie, M. S. Hybertsen, J. B. Neaton, and L. Venkataraman, "Mechanically controled binary conductance switching of a singlemolecule junstion," Nature nanotechnology, vol. 4, pp. 230-234, 2009.

[3] H. Song, Y. Kim, Y. H. Jang, M. A. Reed, and T. Lee, "Observation of molecular orbtal gating," Nature, vol. 462, pp. 1039-1043, 2009.

[4] K. C. Neuman, T. Lionnet, and J.-F. Allemand,

"Single-Moecule

Micromanipulation Techniques," Annual Review of Material Research, vol. 37, pp. 33-67, 2007.

[5] L. Miao and J. M. Seminario, "Molecular Dynamics Simulations of Signal Transmission through a Glycine Peptide Chain," J. Chem. Physics, vol. 127, pp. 134708(1-5), 2007.
[6] J. M. Seminario and L. Yan, "Cascade Configuration of Logical Gates Processing Information Encoded in Molecular Potentials " Int. Journal of Quantum Chemistry, vol. 107, pp. 754-761, 2007.

[7] A. E. Popescu and R. Ionicioiu, "Allelectrical quantum computational with mobile spin qubits," Physical Review B, vol. 69, 2004.

[8] L. Jiang, J. S. Hodges, J. R. Maze, P. Maurer, J. M. Taylor, D. G. Cory, P. R. Hemmer, R. L. Walsworth, A. Yacoby, A. S. Zibrov, and M. D. Lukin, "Repetitive Readout of a Single Electronic Spin via Quantum Logic with Nuclear Spin Ancillae," Science, vol. 326, pp. 267-272, 2009.

[9] B. P. Lanyon, J. D. Whitfield, G. G. Gillet, M. E. Goggin, M. P. Almeida, I. Kassal, J. D. Biamonte, M. Moheseni, B. J. Powell, M. Barbieri, A. Aspuru-Guzik, and A. G. White, "Towards quantum chemistry on a quantum computer," Nautre Chemistry, vol. 2, pp. 106-111, 2010.

[10] D. Englund, I. Fushman, A. Faraon, and J. Vuckovic, "Quatum dots in photonic crystals: From quantum information processing to single photon nonlinear optics," Photonics and Nanostructures-Fundamental and Applications, vol. 7, pp. 56-62, 2009.

[11] D. A. Miller, "Are optical transistors the logical next step?," Nature Photonics, vol. 4, pp. 1-5, 2010.

[12] B. S. Ham and J. Hahn, "Observations of ultraslow light-based phhoton logic gates: NAND/OR," Applied Physics Letters, vol. 94, pp. 101110(1-3), 2009.

[13] F. Walsh, S. Balasubramaniam, D. Botvich, T. Suda, T. Nakano, S. F. Bush, and M. O. Foghlu. (2009). Hybrid DNA and Enzyme Based Computing for Address, Encoding, Link Switching and Error Correction in Molecular Communication. 3.

[14] J. M. Seminario, "Molecular Electronics: Approaching reality," Nature, vol. 4, pp. 111113, 2005.

[15] J. M. Seminario, P. Derosa, L. Cordova, and B. Bozard, "Molecular Dynamics " in Computational Chemistry: Reviews of Current Trends. vol. 9, J. Leszczynski, Ed., ed: World Scientific, 2005, pp. 85-118.

[16] J. M. Seminario, Y. Ma, and V. Tarigopula, "The Nanocell: A Chemically Assemled Molecular Electronic Circuit," IEEE Sensors, vol. 6, pp. 1614-1626, 2006.

[17] A. J. Gimenez, G. Luna-Bárcenas, and J. M. Seminario, "Emulation of Molecular Programmability Using Microelectronics 
Programmable Devices," J. Phys. Chem. C, vol. 113, pp. 16254-16258, 2009.

[18] A. J. Gimenez, G. Luna-Barcenas, and J. M. Seminario, "Analysis of Nano and Molecular Arrays of Negative Differential Resistance Devices for Sensing and Electronics," IEEE Sensors, vol. 9, pp. 1136-1141, 2009.

[19] J. M. Seminario, L. Yan, and Y. Ma, "Scenario for Molecular-Level Signal Processing," IEEE, vol. 93, pp. 1753-1764, 2005.

[20] L. Yan and J. M. Seminario, "Molecular Modeling Toward Molecular Potentials," Int. Journal of Quantum Chemistry, vol. 106, pp. 1964-1969, 2006.

[21] J. M. Seminario, L. Yan, and Y. Ma, "Nanodetectors using Molecular Circuits Operating at $\mathrm{THz}$ Frequencies," Proceedings of SPIE, vol. 5995, pp. 59950R-(1-15), 2005.

[22] N. L. Rangel and J. M. Seminario, "Molecular Electrostatic Potential Devices on Graphite and Silicon Surfaces," J. Phys. Chem. A, vol. 110, pp. 12298-12302, 2006.

[23] N. L. Rangel and J. M. Seminario, "Nanomicrointerfaces to read Molecular Potentials into current-voltage based Electronics," J. Chem. Physics, vol. 128, pp. 114711(1-9), 2008.

[24] L. R. Saenz, P. B. Balbuena, and J. M. Seminario, "Platinum testbeds: Interaction with oxygen," J. Phys. Chem. A, vol. 110, pp. 11968-11974, 2006.

[25] C. Herrera and J. M. Seminario, "Study of nano--Structurated Silicon-Phenyl nanoclusters Towards Single Molecule Sensing," Int. J. High Speed Electron. Syst., vol. 17 pp. 327-338, 2007.

[26] C. Herrera and J. M. Seminario, "Study of Nanostructured Silicon-Phenyl nanoclusters Toward single Molecule Sensing," Int. J. of High Speed Electronics and Systems, vol. 17, pp. 327-338, 2007.

[27] N. L. Rangel and J. M. Seminario, "Graphene Terahertz Generators for Molecular Circuits and Sensors," J. Phys. Chem. A, vol. 112, pp. 13699-13705, 2008.

[28] J. M. Seminario, L. Yan, and Y. Ma, "Encoding and transport of information in molecular and biomolecular systems," Proc. IEEE Nanotech. Conf., vol. 5, pp. 65-68, 2005.

[29] J. M. Seminario, L. Yan, and Y. Ma, "Transmission of Vibronic Signals in Molecular Circuits," J. Phys. Chem. A, vol. 109, pp. 9712-9715, 2005.

[30] J. M. Seminario, L. Yan, and Y. Ma, "Encoding and Transport of Information in
Molecular and Biomolecular Systems," IEEE, vol. 5, pp. 436-440, 2006.

[31] K. Salazar-Salinas, L. A. Jauregui, C. KubliGarfias, and J. M. Seminario, "Molecular Biosensor based on a Coordinated iron complex," J. Chem. Physics, vol. 130, pp. 105101(1-9), 2009.

[32] L. A. Jauregui, K. Salazar-Salinas, and J. M. Seminario, "Transverse Electronic Transport in Double-Stranded DNA Nucleotides," J. Phys. Chem. B, vol. 113, pp. 6230-6239, 2009.

[33] L. A. Jauregui and J. M. Seminario, "A DNA Sensors for Sequencing and Mismatches Based Electron Transport Through WatsonCrick and Non-Watson-Crick Base Pairs," IEEE Sensors, vol. 8, pp. 803-813, 2008.

[34] N. L. Rangel, K. S. Williams, and J. M. Seminario, "Light-Activated Molecular Conductvity of the Photoreaction of Vitamine $\mathrm{D}_{3}, " \mathrm{~J}$. Phys. Chem. A, vol. 113, pp. 6740-6744, 2009. 